\title{
FINITE LINEAR GROUPS WHOSE RING OF INVARIANTS IS A COMPLETE INTERSECTION
}

\author{
BY VICTOR KAC AND KEI-ICHI WATANABE
}

\begin{abstract}
The celebrated Shephard-Todd-Chevalley theorem says that for a finite linear group $\mathrm{G}$ operating on the $n$-dimensional complex vector space the ring $R$ of invariant polynomials is a polynomial ring if and only if $G$ is generated by pseudoreflections $(g \in G$ is a pseudoreflection if $\operatorname{rank}(g-I)=1$ ). In this note we give a simple topological proof of the following statement:

If $R$ has $m$ generators such that their ideal of relations is generated by $m-n+s$ elements, then $G$ is generated by those $g \in G$ such that $\operatorname{rank}(g-n)$ $\leqslant s+2$.

In the case $s=0$ this gives a necessary condition for $R$ to be a complete intersection. Our argument also gives a new simple proof of the "only if" part of the Shephard-Todd-Chevalley theorem in the case of an arbitrary ground field.
\end{abstract}

Let $k$ be a field and let $G$ be a finite subgroup of $G L(n, k)$. The group $G$ acts naturally on the polynomial ring $S=k\left[x_{1}, \ldots, x_{n}\right]$ and we put $R=S^{G}$ to be the invariant subring of $G$. We say that $R$ is a polynomial ring if $R$ is generated by $n$ (algebraically independent) elements, and that $R$ is a complete intersection if $R$ is isomorphic to $k\left[y_{1}, \ldots, y_{n+r}\right] / J$, where $J$ is an ideal generated by $r(=\operatorname{emb} \operatorname{dim} R-\operatorname{dim} R)$ elements. In this paper we prove the following

THEOREM A. If $R$ is a complete intersection, then $G$ is generated by the set $\{g \in G \mid \operatorname{rank}(g-I) \leqslant 2\}$ (where $I$ is the identity matrix).

The proof is based on two simple topological lemmas. We can assume that the ground field $k$ is algebraically closed.

Let $f: \operatorname{Spec}(S) \rightarrow \operatorname{Spec}(R)$ be the quotient morphism. Let $X^{\prime}$ and $Y^{\prime}$ be the henselisations of $\operatorname{Spec}(S)$ at 0 and of $\operatorname{Spec}(R)$ at $f(0)$ respectively and $f^{\prime}$ : $X^{\prime} \rightarrow Y^{\prime}$ the associated morphism. Then the action of $G$ on $\operatorname{Spec}(S)$ lifts to $X^{\prime}$ and $f^{\prime}$ is the quotient morphism. We use henselisations in order to deal with simply connected (i.e. without nontrivial étale coverings) schemes $X^{\prime}$ and $Y^{\prime}$. If char $k=0$, then $\operatorname{Spec}(S)$ and $\operatorname{Spec}(R)$ are simply connected and the henselisation is not necessary.

LEMMA 1. Let $Y^{\prime}$ be a simply connected scheme, $Z$ a closed subscheme and $Y=Y^{\prime}-Z$. If $Y^{\prime}$ is a complete intersection and $\operatorname{codim} Z \geqslant 3$, then $Y$ is simply connected.

Proof. The proof follows from [2, X, 3.3 and 3.4].

Received by the editors August 4, 1981.

1980 Mathematics Subject Classification. Primary 14D25; Secondary 14L30. 
REMARK 1. The conclusion of Lemma 1 holds if instead of $Y$ to be a complete intersection and $\operatorname{codim} Z \geqslant 3$, we require that $Y$ is regular and $\operatorname{codim} Z \geqslant 2$.

Lemma 2 (VInBerg). Let $X$ be an integral scheme and $G$ a finite subgroup of $\operatorname{Aut}_{k}(X)$. Let $Y=X / G$ and $f: X \rightarrow Y$ be the quotient morphism. For a closed point $x$ of $X$ let $G_{x}$ denote the stabilizer of $x$. If $Y$ is simply connected, then $G$ is generated by all $G_{x}$ 's.

Proof. Let $H$ be the subgroup of $G$ generated by all $G_{x}$ 's; it is a normal subgroup. Then for the action of $G / H$ on $X / H$ any $g \neq e$ has no closed fixed points and by $[1, \mathrm{I}, 10.11]$, the morphism $X / H \rightarrow Y$ is an étale covering. By our assumption, we have $G=H$.

Proof of Theorem A. For $g \in G$ let $L_{g}$ denote the subscheme of fixed points of $g$ on $X^{\prime}$. Let $L$ be the union of all $L_{g}$ 's with codim $L_{g} \geqslant 3$, and put $X=X^{\prime}-L, Z=f^{\prime}(L)$ and $Y=Y^{\prime}-Z$. Note that $Y^{\prime}$ is a complete intersection since $\operatorname{Spec}(R)$ is, and $Z$ is a closed subscheme in $Y^{\prime}$ of codimension $\geqslant 3$. Furthermore, $X$ is an integral scheme with the induced $G$-action, $Y=X / G$, and $Y$ is simply connected by Lemma 1 . Hence, by Lemma $2, G$ is generated by all $G_{x}$ 's, $x \in X$. But by the definition of $X, g \in G_{x}$ for some $x \in X$ if and only if codim $L_{g} \leqslant 2$ or, equivalently, $\operatorname{rank}(g-I) \leqslant 2$.

REMARK 2. $R$ is a complete intersection for any $G \subset G L(2, \mathrm{C})$ (F. Klein). It is not difficult to construct an example of a finite group $G \subset \operatorname{SL}(3, \mathrm{C})$ generated by two matrices $A_{1}$ and $A_{2}$, such that $\operatorname{rank}\left(A_{i}-I\right)=2, i=1,2$, but $R$ is not a complete intersection [7].

REMARK 3. Our argument together with Remark 1 gives a short topological proof of the "only if" part of the Shephard-Todd-Chevalley theorem [3, 5] over any ground field $k$ : If $R$ is a polynomial ring, then $G$ is generated by pseudoreflections. It is not difficult to show that, furthermore, $G_{x}$ is generated by pseudoreflections for any $x$. The first author takes this opportunity to suggest the following risky conjecture: Conversely, if $G_{x}$ is generated by pseudoreflections for any $x$, then $R$ is a polynomial ring.

If the ground field is the field $\mathbf{C}$ of complex numbers, the topology of $\operatorname{Spec}(R)$ is better known and we can prove the following more general theorem.

THEOREM B. Let $G$ be a finite subgroup of $G L(n, \mathrm{C})$ and $S=\mathrm{C}\left[x_{1}, \ldots, x_{n}\right]$. If $R=S^{G}$ has $m$ generators such that their ideal of relations is generated by $m-n+s$ elements, then $G$ is generated by those $g \in G$ such that $\operatorname{rank}(g-I) \leqslant$ $s+2$. 
Proof. We set $X^{\prime}=\operatorname{Spec}(S), Y^{\prime}=\operatorname{Spec}(R)$. By the same argument as above, we have only to prove that $Y=Y^{\prime}-Z$ is simply connected if $\operatorname{codim} Z \leqslant$ $s+3$, under our assumption. The corresponding generalisation of Lemma 1 in the complex case has been recently proved by Goresky and Macpherson [4] .

REMARK 4. We do not know whether Theorem B is true for an arbitrary ground field.

Note, finally, that we can strengthen Theorem A (and in a similar way, Theorem B) as follows (cf. Remark 3).

THEOREM C. If $R$ is a complete intersection, then each $G_{x}$ is generated by $\left\{g \in G_{x} \mid \operatorname{rank}(g-I) \leqslant 2\right\}$.

Proof. Let $X=\operatorname{Spec}(S), Y=X / G_{x}$ and denote by $\pi: X \rightarrow Y$ the quotient morphism. Then the morphism $Y \rightarrow X / G$ is étale at $\pi(x)$ by $[1, \mathrm{I}, 10.11]$, Hence the local ring at $\pi(x) \in Y$ is a complete intersection, and we can apply Theorem A.

REMARK 5. The converse of Theorem $\mathrm{C}$ is false (cf. Remark 2).

The authors are grateful to the organizers of the meeting in Trento, Italy (June 1981), where the authors met, for their hospitality, especially to C. Procesi who also suggested to combine the topological argument of the first author and a conjecture of the second author. Also, the authors are thankful to P. Deligne who suggested to use henselisation, and to M. Goresky and R. Macpherson who showed us the proof of the fact used to prove Theorem B. The first author thanks B. Weisfeiler for debates concerning the conjecture in Remark 3 and gratefully acknowledges the support of IHES.

\section{REFERENCES}

1. A. Grothendieck, Revetements étales et groupes fondamental (SGA, 1), Lecture Notes in Math., vol. 224, Springer-Verlag, Berlin and New York, 1971.

2. Cohomologie locale des faisceaux coherents et théorèmes des Lefschetz locaux et globaux (SGA, 2), North-Holland, Amsterdam, 1968.

3. C. Chevalley, Invariants of finite groups generated by reflections, Amer. J. Math. 67 (1955), 778-782.

4. M. Goresky, Letter to the first author, June 1981. 274-304.

5. G. G. Shephard and J. A. Todd, Finite reflection groups, Canad. J. Math. 6 (1954),

6. K.-i. Watanabe, Invariant subrings of finite groups which are complete intersections. I. Invariant subrings of finite Abelian groups, Nagoya Math. J. 77 (1980), 89-98.

7. K.-i. Watanabe and D. Rotillon, Invariant subrings of $C[X, Y, Z]$ which are complete intersections (in preparation).

DEPARTMENT OF MATHEMATICS, MASSACHUSETTS INSTITUTE OF TECHNOLOGY, CAMBRIDGE, MASSACHUSETTS 02139

DEPARTMENT OF MATHEMATICS, NAGOYA INSTITUTE OF TECHNOLOGY, GOKISO-CHO, SHOWA-KY, NAGOYA, 466, JAPAN 